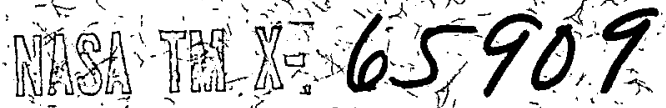

\section{THE INFLUENCE OF LINE SHAPE AND BAND STRUCIURE ON TEMPERATURES IN PLANETARY ATMOSPHERES}

(NASA-TM-X-6509)

SHAPE AND BAND STRUCTURE ON TEMPERATURES IN

PLANETARY ATMOSPHERES A. Arking, et al

(NASA) Jun, 197247 p

ALBERT AR KING

A
(5) TH THE I
MOSPHERES
$197247 \mathrm{p}$

KENNETH GROSSMAN

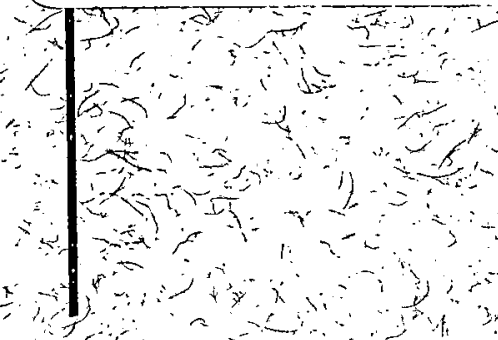

\section{JUNE 1972}

\section{GSFC}

GODDARD SPACE FLIGHT CENTER

GREENBELT, MARYLAND

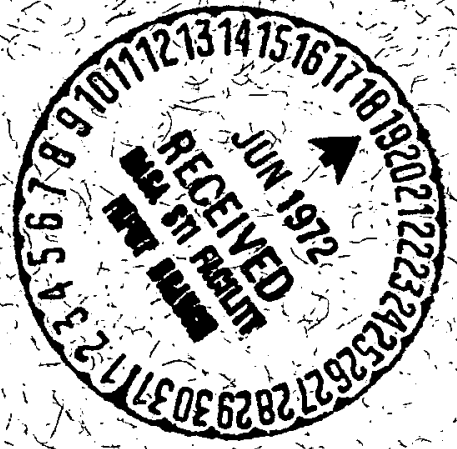


$\mathrm{X}-651-72-186$

Preprint

THE INFLUENCE OF LINE SHAPE AND BAND STRUCTURE ON TEMPERATURES IN PLANETARY ATMOSPHERES

\author{
Albert Arking \\ Goddard Space Flight Center \\ Greenbelt, Maryland \\ Kenneth Grossman \\ Computer Sciences Corporation \\ New York, New York
}

June 1972

GODDARD SPACE FLIGHT CENTER

Greenbelt, Maryland 


\title{
THE INFLUENCE OF LINE SHAPE AND BAND STRUCTURE \\ - \\ ON TEMPERATURES IN PLANETARY ATMOSPHERES
}

\author{
Albert Arking \\ Goddard Space Flight Center \\ Greenbelt, Maryland \\ Kenneth Grossman. \\ Computer Sciences Corporation \\ New York, New York
}

\section{ABSTRACT}

Numerical experiments are performed to examine the effects of line shape and band structure on the radiative equilibrium temperature profile in planetary atmospheres. In order to accurately determine these effects, a method for calculating radiative terms is developed, which avoids the usual approximations. It differs from the more commonly used methods in that it allows arbitrary dependence of the absorption coefficient on wave number, without requiring tedious line by line integration and without the constraints of band models. The present formulation is restricted to homogeneous atmospheres but the concept can be extended to the more general case. The numerical experiments reveal that the line shape and band structure of the absorbing gases have a large effect on temperatures in the higher layers of the atmosphere (corresponding to the stratosphere and mesosphere). The more non-grey the spectrum - i.e., the higher the peaks and the deeper the troughs in the spectrum - the lower the temperature. 


\section{THE INFLUENCE OF LINE SHAPE AND BAND STRUCTURE ON TEMPERATURES IN PLANETARY ATMOSPHERES}

1. Introduction

Theoretical studies have shown that radiative exchange plays an important role in the structure and general circulation of the stratosphere and mesosphere. (See, for example, Murgatroyd and Goody (1958); Leovy (1964); Kuhn and London (1969)). In the Mars atmosphere, radiation may even play a dominant role (Goody and Belton (1967)). Because of the complexity of molecular absorption and emission processes, however, all calculations are based upon approximate treatment of various aspects of the radiative transfer problem. The effects of these approximations, especially in the calculation of transmission functions, have not been adequately explored. The purpose of this paper is twofold: 1) to describe a method for calculating transmission functions and radiative terms in which the approximations are eliminated, or at least subject to control; 2) to study the effects of line shape and band structure on radiative equilibrium temperatures in planetary atmospheres.

The approximations usually made in radiative transfer problems fall into four categories:

1. Numerical approximations in replacing integration over height by a quadrature formula.

2. Treatment of the angular distribution of the vertical flux.

3. Transmission function calculations for a given composition, temperature, and pressure. 


\section{Transmission functions over inhomogeneous paths.}

Numerical approximations are unavoidable due to the nature of the problem and the limitations of our computational tools. The errors, however, are subject to control and in the end, one balances the desired accuracy against the computational costs.

The last three categories of approximations represent more fundamental obstacles. Our lack of knowledge concerning molecular absorption and emission, combined with the enormous complexity of infrared spectra, have inhibited attempts to do accurate calculations. The angular distribution of vertical flux is usually taken into account by the use of a diffusivity factor (see, e.g., Goody, 1964, sec. 6.2; also, Kondratyev, 1969, sec. 1.3). This approximation is satisfactory for paths of optical thickness $\sim 1$ but leads to substantial errors for longer paths. In the methods and calculations presented here this approximation is not necessary; the angular distribution of the vertical flux is fully taken into account.

Until recent years, the lack of data on high resolution infrared spectra of atmospheric gases has made it necessary to use laboratory measured transmission functions. Their principal drawback is the limited range of pressure, temperature, and absorber thickness for which measurements are available. To fill the gap, theoretical band models were developed, based upon highly idealized representations of the distribution of lines within a spectral interval. The parameters for the band models were chosen to obtain the best fit to the laboratory measurements. Although it is not always recognized in the literature, the band models are little more than 
interpolation formulas, whose validity is restricted to the range of conditions and absorber thickness within which the band models agree with laboratory measurements. Extrapolation beyond this range can lead to appreciable errors.

During the last decade an enormous effort has gone into determining fundamental molecular properties from which the positions, intensities, and halfwidths of spectral lines for several atmospheric gases have been compiled (see, e.g., Drayson and Young (1967), Benedict and Calfee (1967)). With suitable assumptions concerning the line shape, one can calculate from these data the absorption coefficient versus wave number, $k(\nu)$, for a given temperature and pressure.

In principle, one can directly employ $k(\nu)$ in the radiative transfer equations and obtain numerical solutions. The only drawback is the complicated nature of the function $k(\nu)$. A typical band will contain anywhere from several hundred to several thousand lines that must be included to obtain reasonable accuracy. The half-widths are usually sufficiently smaller than the line spacing that $k$ becomes a rapidly varying function of $\nu$. As a result, integration over $\nu$ (line by line) becomes a time consuming and costly chore.

For homogeneous atmospheres, where the line parameters are assumed to be independent of height, one can achieve the same results as line by line integration, but in a much simpler way. In its present application, the method emerged from a private discussion with H. C. van de Hulst and E. Spiegel in 1963 and was presented by Arking (1968). The basic idea, however, is quite old. It is described in a treatise on atmospheric radiation by Kondratyev (1969) with reference to a 1939 Russian 
paper by A. I. Lebedinsky. It has also seen recent use in calculating line-blanketing effects in stellar atmospheres (Strom and Kurucz, 1966).

The method makes use of the fact that for a homogeneous atmosphere, the transmission within a relatively wide spectral interval is independent of the ordering of the value of $\mathrm{k}$ with respect to $\nu$, but depends upon the fraction of the interval that is associated with a particular value of $k$. This latter quantity is the distribution of $k$-values within the spectral interval and is referred to as the k-distribution. function.

Use of the k-distribution function considerably simplifies the calculation of transmission functions for homogeneous atmospheric layers. In the calculations presented below, it is used to determine radiative equilibrium temperatures in artificial atmospheres with highly idealized forms of $k(\nu)$, but it is equally applicable to real atmospheres, provided one has the means to generate $k(\nu)$.

The last remaining approximation in calculating radiative terms is to take full account of inhomogeneity in the atmosphere. This introduces a new dimension of complexity into the problem. If the atmosphere is horizontally homogeneous, one can extend the method of the $k$-distribution function by defining a separate $k-$ distribution function for each layer in the atmosphere. Such a method is feasible only if one severely restricts the number of levels in the atmosphere or if one can find a way to parametrize the dependence of the k-distribution function on height. The problem of inhomogeneous atmospheres will be deferred to a later study. 
The method is applied below to the problem of calculating the vertical temperature profile in a planetary atmosphere under conditions of radiative equilibrium. Numerical solutions to the resulting equations are obtained by a generalized Newton-Raphson scheme, which converges quadratically with each iteration. The atmosphere is assumed to be homogeneous (horizontally and vertically). The absorption versus wave number is permitted to take one of a number of highly idealized forms and the calculated temperature profiles are compared. The results reveal that the temperatures near the top of the atmosphere (corresponding to the stratosphere and mesosphere) are sensitive to the detailed characteristics of the infrared spectrum of the absorbing gases.

The assumptions of the model and the resulting atmospheric structure equations are presented in section 2. The k-distribution functions and transmission functions are calculated in section 3. The method of solution of the equations is described in section 4. The numerical results are discussed in section 5. A summary is presented in section 6. 
2. Equations of Atmospheric Structure.

We make the following assumptions:

1. Steady state conditions.

2. The atmosphere is plane parallel and uniform in the horizontal plane.

3. Radiation is the only means of energy transport and it obeys Kirchoff's Law--i.e., the ratio of the spontaneous emission coefficient to the absorption coefficient (already corrected for induced emission) is equal to the Planck function

$$
B_{\nu}(T)=\frac{2 h c \nu^{3}}{e^{h c \nu / k T}-1}
$$

where $T$ denotes temperature and $\nu$ denotes wave number.

The vertical parameter will be the "mass level", denoted by $p$ and defined as the mass per unit area above a given level in the atmosphere. (In an atmosphere in hydrostatic equilibrium, it is approximately the pressure divided by the acceleration of gravity.) The object of the calculation is to obtain the vertical temperature profile, $T(p)$, under the above assumptions.

The conservation of energy at each level requires

$$
4 \pi \int_{0}^{\infty} k_{\nu} B_{\nu}(T(p)) d \nu=4 \pi \int_{0}^{\infty} k_{\nu} \bar{I}_{\nu}(p) d v+Q(p)
$$

where $k_{\nu}$ is the mass absorption coefficient at wave number $\nu, Q(p)$ is the heating rate per unit mass due to solar radiation, and $\bar{I}_{\nu}$ is the specific intensity of infrared radiation, $I_{\nu}$, averaged over all directions

$$
\bar{I}_{\nu}(p)=\frac{1}{4 \pi} \int_{0}^{2 \pi} \int_{-1}^{1} I_{\nu}(p, \mu, \varphi) d \mu d \varphi
$$


where $\mu$ is the cosine of the solar zenith angle and $\varphi$ is the corresponding azimuth angle. The term on the left side of Eq. (1) represents infrared radiation emitted by the atmosphere; the first term on the right, the absorbed infrared radiation; the second term on the right, the absorbed solar radiation.

The specific intensity is obtained from a solution to the radiative transfer equation

$$
\mu \frac{d I_{\nu}}{d p}=k_{\nu} I_{\nu}-k_{\nu} B_{\nu}(T)
$$

with the following boundary conditions: no downward radiation at the top of the atmosphere

$$
I_{\nu}(0, \mu)=0 \quad \mu<0
$$

and a blackbody surface at temperature $T_{s}$ (to be determined below) at the bottom of the atmosphere

$$
I_{\nu}\left(p_{s}, \mu\right)=B_{\nu}\left(T_{s}\right) \quad \mu>0
$$

where $p_{s}$ is the atmospheric mass above the planetary surface. Solutions to Eq. (2) are found in standard textbooks on radiative transfer (see, for example, Kourganoff, 1952).

With the boundary conditions given above, the solution is averaged over $4 \pi$ steradians and inserted into Eq. (1) to yield

$$
\begin{aligned}
& 2 \pi \int_{0}^{\infty} k_{\nu}(p)\left\{2 B_{\nu}(T(p))-B_{\nu}\left(T_{s}\right) E_{2}\left(\int_{p}^{p_{s}} k_{\nu}\left(p^{\prime}\right) d p^{\prime}\right)\right. \\
& -\int_{p}^{p_{s}} B_{\nu}\left(T\left(p^{\prime \prime}\right)\right) E_{1}\left(\int_{p}^{p^{\prime \prime}} k_{\nu}\left(p^{\prime}\right) d p^{\prime}\right) k_{\nu}\left(p^{\prime \prime}\right) d p^{\prime \prime} \\
& \left.-\int_{0}^{p} B_{\nu}\left(T\left(p^{\prime \prime}\right)\right) E_{1}\left(\int_{p^{\prime \prime}}^{p} k_{\nu}\left(p^{\prime}\right) d p^{\prime}\right) k_{\nu}\left(p^{\prime \prime}\right) d p^{\prime \prime}\right\} d \nu=Q(p)
\end{aligned}
$$

where the dependence of $k_{\nu}$ on $p$ is explicitly indicated and 
where $E_{n}(x)$ is the exponential integral

$$
E_{n}(x)=\int_{0}^{1} e^{-\frac{x}{\mu}} \mu^{n-2} d \mu
$$

In addition to the temperature profile $T(p)$, the surface temperature

$\mathrm{T}_{s}$ is an unknown quantity, but it can be obtained simultaneously with the temperature profile by applying energy balance considerations to the surfaceatmosphere interface

$$
\pi B_{s}=\sigma T_{e}^{4}-\int_{0}^{p_{s}} Q(p) d p+\int_{0}^{\infty} 2 \pi \int_{-1}^{0} I_{\nu}\left(p_{s}, \mu\right) \mu d \mu d \nu
$$

where $\pi B_{s} \equiv \sigma T_{s}^{4}$ is the infrared radiative flux emitted by the planetary surface; $\sigma T_{e}^{4}$ is the net solar radiative flux absorbed by the surface and atmosphere together (i.e. the solar flux incident at the top of the atmosphere minus what is reflected back to space) thereby defining the "effective temperature" of the planet, $T_{e}$; $\sigma$ is the Stefan-Boltzmann constant. The second term on the right is that part of the net solar radiative flux that is absorbed by the atmosphere and the last term is the atmospheric infrared flux incident on the planetary surface.

Substituting the solution of Eq. (2) into Eq. (4) yields

$$
\begin{gathered}
\pi B_{s}=\sigma T_{e}^{4}-\int_{0}^{P_{s}} Q(p) d p+2 \pi \int_{0}^{\infty} \int_{0}^{P_{s}} B_{\nu}(T(p)) \\
\cdot E_{2}\left(\int_{p}^{P_{s}} k_{\nu}\left(p^{\prime}\right) d p^{\prime}\right) k_{\nu}(p) d p d \nu
\end{gathered}
$$

The wave number range of interest--i.e., where $B_{\nu}(T)$ is appreciably different from zero--extends from about 100 to $2500 \mathrm{~cm}^{-1}$ for Earth atmospheric temperatures. In this range $B_{\nu}(T)$ is a smoothly varying function with a single maximum (in the 
vicinity of $500 \mathrm{~cm}^{-1}$ for Earth). In contrast, $k_{v}$ is a rapidly varying function whose structure depends upon composition, pressure, and temperature. Under typical conditions, the details in $k_{\nu}$ require a resolution in space of $\sim 10^{-3} \mathrm{~cm}^{-1}$ or less. To avoid the storage and manipulation of such large amounts of data, one works instead with transmission functions which are pre-computed for a set of wave number intervals spanning the spectrum. The intervals in $v$-space are suitably chosen so that the variation of $B_{\nu}(T)$ within the interval can be neglected and the terms containing $k_{\nu}$ can be replaced by mean values within the interval. Dividing $\nu$-space into $m$ intervals, with the $i^{\text {th }}$ interval extending from $\nu_{i-1}$ to $\nu_{i}$, and the concatenation of the $m$ intervals extending from $\nu_{0}=0$ to $\nu_{m}=\infty$, the transmission functions are defined as follows:

Intensity transmission

$$
\bar{\tau}_{i}\left(p_{1}, p_{2}\right)=\frac{1}{\nu_{i}-\nu} \int_{i-1}^{\nu_{i}} e^{-\int_{p_{1}}^{p_{2}} k_{\nu}(p) d p} d \nu \quad p_{1} \leqslant p_{2}
$$

Isotropic flux transmission and its derivates

$$
\begin{aligned}
\tau_{i}\left(p_{1}, p_{3}\right) & =2 \int_{0}^{1} \tau_{i}\left(\frac{p_{1}}{\mu}, \frac{p_{2}}{\mu}\right) \mu d \mu \\
& =\frac{2}{\nu_{i}-\nu} \int_{i-1}^{\nu} \nu_{i-1} E_{3}\left(\int_{p_{1}}^{p_{2}} k_{\nu}(p) d p\right) d \nu \\
\tau_{i}{ }^{10}\left(p_{1}, p_{2}\right) & =\frac{d}{d p_{1}} \tau_{i}\left(p_{1}, p_{2}\right)
\end{aligned}
$$




$$
\begin{aligned}
& \tau_{i}^{0 i}\left(p_{1}, p_{3}\right)=-\frac{d}{d p_{2}} \tau_{i}\left(p_{1}, p_{2}\right) \\
& \tau_{i}^{11}\left(p_{1}, p_{2}\right)=-\frac{d^{2}}{d p_{1} d p_{2}} \tau_{i}\left(p_{1}, p_{2}\right)
\end{aligned}
$$

The intensity transmission is what one usually measures in a laboratory beam experiment. The isotropic flux transmission is the fraction of the radiative flux from an isotropic source which is transmitted through the layer $\left(p_{1}, p_{3}\right)$. Of course; the transmitted flux is no longer isotropic and the multiplicative property of transmission functions, which holds for the intensity transmission under a wide variety of conditions (see, for example, Goody, 1964, Sec. 4.1) does not apply, strictly speaking, to the isotropic flux transmission.

The derivatives of the transmisslon functions appear in the atmospheric structure equations, (3) and (5), which can now be written:

$$
\begin{aligned}
& \pi \sum_{i=1}^{m}\left\{4 \bar{k}_{i}(p) B_{i}(p)-B_{s i} \tau_{i}{ }^{10}\left(p, p_{s}\right)-\int_{p}^{p_{s}} B_{i}\left(p^{\prime}\right) \tau_{i}{ }^{11}\left(p, p^{\prime}\right) d p^{\prime}\right. \\
& \left.\quad-\int_{0}^{p} B_{i}\left(p^{\prime}\right) \tau_{i}{ }^{11}\left(p^{\prime}, p\right) d p^{\prime}\right\}=Q(p) \\
& \pi B_{s}=\sigma T_{e}{ }^{4}-\int_{0}^{p_{s}} Q(p) d p+\pi \sum_{i=1}^{m} \int_{0}^{p_{s}} B_{i}(p) \tau_{i}{ }^{10}\left(p, p_{s}\right) d p
\end{aligned}
$$

where we have defined

$$
\begin{array}{ll}
B_{i}(p)=\int_{\nu}^{\nu i-i} B_{\nu}(T(p)) d \nu & 1 \leq i \leq m \\
B_{s i}=\int_{\nu}^{\nu} B_{\nu}\left(T_{s}\right) d \nu & 1 \leq i \leq m
\end{array}
$$




$$
\bar{k}_{i}(p)=\frac{1}{\nu_{i}-\nu_{i}-1} \int_{\nu_{i}-1}^{\nu_{i}} k_{\nu}(p) d \nu=\frac{1}{4}\left[\tau_{i}^{10}(p, p)-\tau_{i}^{01}(p, p)\right]
$$

The vertical temperature profile and planetary surface temperature can now be calculated from Eqs. (11) and (12), subject to the prior determination of the transmission functions which are in themselves dependent upon pressure and temperature.

\section{Transmission Functions.}

In this paper we restrict our considerations to homogeneous atmospheres, where the absorption coefficient is assumed independent of pressure and temperature. In that case, Eqs. (6) through (10) reveal that it is only the distribution of $k$-values within the interval which determines the transmission, the ordering of the values having no bearing whatsoever.

We can, therefore, proceed to calculate the transmission functions using distribution functions, $h_{i}(k)$, which are normalized to unity, to represent the frequency of occurrence of absorption coefficient value $k$ within the $i^{\text {th }}$ wave number interval. The transmission functions can be written in terms of $h_{i}(k)$ by replacing 


$$
\frac{1}{\nu_{i}-\nu_{i}-1} \int_{\nu_{i-1}}^{\nu_{i}} \ldots d v \rightarrow \int_{0}^{\infty} \ldots h_{i}(k) d k
$$

and noting that $k_{\nu}$ or $k$ is independent of $p$.

Thus,

$$
\begin{aligned}
& \bar{\tau}_{i}\left(p_{1}, p_{2}\right)=\int_{0}^{\infty} e^{-k\left(p_{2}-p_{1}\right)_{h_{i}}(k) d k} \\
& \tau_{i}\left(p_{1}, p_{2}\right)=2 \int_{0}^{\infty} E_{3}\left(k\left|p_{2}-p_{1}\right|\right) h_{i}(k) d k \\
& \tau_{i}^{10}\left(p_{1}, p_{2}\right)=\tau_{i}^{01}\left(p_{1}, p_{2}\right)=2 \int_{0}^{\infty} E_{2}\left(k\left|p_{2}-p_{1}\right|\right) k h_{i}(k) d k \\
& \tau_{i}^{11}\left(p_{1} p_{2}\right)=2 \int_{0}^{\infty} E_{1}\left(k\left|p_{2}-p_{1}\right|\right) k^{2} h_{i}(k) d k
\end{aligned}
$$

Given the function $k_{\nu}$ within a wave number interval $\left(v_{1}, v_{3}\right)$, the $k$-distribution function can be calculated as follows. Divide the interval $\left(\nu_{1}, \nu_{2}\right)$ at all local minima and maxima of $k_{\nu}$ so as to have sub-intervals over which $k_{\nu}$ is monotonic. Label the sub-intervals $1=1,2, \ldots . L$ and denote the length of the $1^{\text {th }}$ sub-interval by $\Delta \nu_{1}$. Within each sub-interval, invert the function $k_{\nu}$ and denote it by $\nu_{1}(k)$. The k-distribution function is then given by

$$
h(k)=\frac{1}{v_{2}-v_{I}} \sum_{I=1}^{L}\left|\frac{d v_{1}}{d k}\right| .
$$

In practice, one can obtain the k-distribution function numerically by a simple statistical procedure: Evaluate $k_{\nu}$ at a number of points chosen at random within the $\left(\nu_{1}, v_{2}\right)$ interval and calculate the frequency distribution of the $k$-values. The accuracy of the $h(k)$, determined in this way, depends upon the number of points in the sample. For complicated $k_{v}$ functions, such as are 
found in the spectra of infrared active gases, this method can result in an enormous saving of computer time, compared to line by line integration.

The k-distribution functions for some highly idealized cases, in which $h(k)$ takes on particularly simple forms, are presented below, followed by an example showing the k-distribution for a portion of the $\mathrm{CO}_{2} 15 \mu$ band.

\section{A. Grey case (GR)}

$k_{\nu}=\bar{k}$ independent of $v_{i}$ the corresponding $k$-distribution is given by

$$
h(k)=\delta(k-\bar{k})
$$

where $\delta(k)$ is the Dirac delta function with normalization $\int_{0}^{\infty} \delta(k) d k=1$.

\section{B. Regular Band of Non-overlapping Lines}

The spectrum is constructed from a series of identical, non-overlapping line profiles, equally spaced, with separation d along the $v$-axis. The line shapes to be considered are square (SQ), triangular (TR), Lorentz (LO) and Doppler (DO). A characteristic half-width is defined for each shape and its ratio to the line spacing is denoted by $\alpha$. The square and triangular profiles have finite widths less than or equal to the line spacing; between the lines,we assume a background absorption level with the value $k_{1}$; at the center of each line, the absorption has a maximum value $k_{2}$. For the Lorentz and Doppler shapes, the profile of each line is cut off at the points 
midway between the peaks so that there is no overlapping; the absorption at the peaks is $k_{2}$ and the minimum absorption, at the cut-off points, is $k_{1}$.

The absorption coefficient, the corresponding $k$-distribution function and the mean absorption are described below for each line shape. $k_{v}$ can be specified in each case by giving the value of $k_{v}$ over an interval $d$ corresponding to the separation between lines, but if we assume the line is centered at $\nu=0$ and take the symmetry of the line shape into account then it is sufficient to specify $k_{v}$ only in the range $0 \leq \nu \leq \frac{d}{2}$.

For the various line shapes, we have

SQ:

$$
\begin{aligned}
k_{\nu}=\left\{\begin{array}{lc}
k_{2} & 0 \leq \nu \leq a d \\
k_{1} & a d<\nu \leq d / 2
\end{array}\right. \\
h(k)=(1-2 a) \delta\left(k-k_{1}\right)+2 \alpha \delta\left(k-k_{a}\right) \\
\bar{k}=k_{1}+2 a\left(k_{3}-k_{1}\right)
\end{aligned}
$$

$T R:$

$$
\begin{gathered}
k_{\nu}=\left\{\begin{array}{cc}
k_{2}-\frac{\nu}{2 a d}\left(k_{2}-k_{1}\right) & 0 \leq \nu \leq 2 a d \\
k_{1} & 2 a d<\nu \leq d / 2
\end{array}\right. \\
h(k)=(1-4 a) \delta\left(k-k_{1}\right)+\left\{\begin{array}{cc}
\frac{4 a}{k_{2}-k_{1}} & k_{1} \leq k \leq k_{3} \\
0 & k<k_{1} \text { or } k>k_{2}
\end{array}\right. \\
\bar{k}=k_{1}+2 a\left(k_{3}-k_{1}\right)
\end{gathered}
$$


LO:

$$
\begin{array}{cc}
k_{\nu}=\frac{k_{a}}{1+(\nu / a d)^{2}} & 0 \leq \nu \leq d / 2 \\
h(k)=\frac{k_{a} \alpha}{k^{3 / 2}\left(k_{2}-k\right)^{\frac{1}{2}}} & k_{1} \leq k \leq k_{2} \\
\bar{k}=2 \alpha k_{2} \tan ^{-1} \frac{1}{2 a} & \\
k_{1}=\frac{k_{2}}{1+\left(\frac{1}{2 a}\right)^{2}} &
\end{array}
$$

DO:

$$
\begin{array}{cc}
k_{\nu}=k_{2} e^{-\left(\frac{\nu}{a d}\right)^{2}} & 0 \leq \nu \leq d / 2 \\
h(k)=\frac{a}{k\left(\ln k_{a} / k\right)^{\frac{1}{2}}} & k_{1} \leq k \leq k_{3} \\
\bar{k}=\sqrt{\pi} a k_{2} \operatorname{erf}\left(\frac{1}{2 a}\right) & \\
k_{1}=k_{2} e^{-\left(\frac{1}{2 a}\right)^{2}}
\end{array}
$$

C. Regular Barid of Overlapping Lines

Here we consider equally spaced Lorentz or Doppler line profiles which are permitted to overlap. The absorption coefficient is obtained by summing the contribution of each line, extending from $-\infty$ to $+\infty$. With Lorentz profiles, referred to as the Elsasser band model, the resulting function has an analytic form (see, for example, 
Goody, 1964, sec. 4.4.1). For the Elsasser model, the absorption coefficient, the corresponding $k$-distribution function, and the mean absorption are expressed either in terms of $k_{2}$, the value at the peaks, and the minimum value $k_{1}$, midway between the peaks, or in terms of $k_{2}$ and $\alpha$, as follows:

EL:

$$
\begin{array}{rlr}
k_{\nu} & =k_{2} \frac{\cosh 2 \pi \alpha-1}{\cosh 2 \pi \alpha-\cos 2 \pi \nu / d} & \\
& =\frac{2 k_{1} k_{2}}{k_{1}+k_{2}-\left(k_{2}-k_{1}\right) \cos 2 \pi \nu / d} & 0 \leq \nu \leq d / 2 \\
h(k) & =\frac{1}{\pi k}\left[\frac{k_{1} k_{3}}{\left(k_{2}-k\right)\left(k-k_{1}\right)}\right]^{\frac{1}{2}} & \\
\bar{k} & =\left(k_{1} k_{2}\right)^{\frac{1}{2}}=k_{2} \tanh \pi \alpha k_{2} \\
k_{1} & =k_{2} \tanh ^{2} \pi \alpha
\end{array}
$$

For Doppler line profiles, $h(k)$ does not hove a closed form but can be obtained numerically by the procedure which led to Eq. (14). The appropriate functions and related parameters can be expressed as follows:

DR:

$$
\begin{aligned}
& k_{\nu}=k_{3} \frac{\sum_{n=-\infty}^{\infty} e^{-\left(\frac{\nu-n d}{\alpha d}\right)^{2}}}{1+2 \sum_{n=1}^{\infty} e^{-\left(\frac{n}{\alpha}\right)^{2}}} \quad 0 \leq \nu \leq d / 2 \\
& \bar{k}=\frac{\sqrt{\pi} \alpha k_{2}}{1+2 \sum_{n=1}^{\infty} e^{-\left(\frac{n}{\alpha}\right)^{2}}}
\end{aligned}
$$




$$
k_{1}=\frac{2 \bar{k}}{\sqrt{\pi} \alpha} \sum_{n=1}^{\infty} e^{-\left(\frac{n-\frac{1}{2}}{\alpha}\right)^{2}}
$$

D. Randomly Distributed Lines

The k-distribution function for lines which are randomly distributed, allowing overlap, can be calculated from the $k$-distribution function for individual lines by applying probability theory.

A particularly simple case of randomly distributed lines occurs when the line shapes are square. For example, if one line contributes an absorption $k_{0}$ over an interval $2 a d$, where $d$ is the mean line spacing, then the permitted values are $k=n k_{0}$, where $n$ is a positive integer, and the k-distribution function is the Poisson distribution

$$
h_{R}\left(k=n k_{0}\right)=\frac{(2 \alpha)^{n} e^{-2 \alpha}}{n !} \quad n=0,1,2 \ldots
$$

with

$$
\bar{k}=2 a k_{0}
$$

For small values of $\alpha$, there would be little difference between randomly or regularly spaced lines and, indeed, the above expression for randomly distributed squares approaches the expression given above for regularly spaced squares as $\alpha \rightarrow 0$.

The general expression for randomly distributed lines of arbitrary shape is a compound Poisson distribution (see, for example, Feller, 1950, chap. XII). 
If $h(k)$ is the $k$-distribution function for an individual line defined over a finite wave number interval, $s$, then the $k$-distribution function for a random distribution of lines with mean spacing $d$, is given by

$$
h_{R}(k)=e^{-\lambda} \sum_{n=0}^{\infty} \frac{\lambda^{n}}{n !} C_{k}\{h\}^{n}
$$

where $\lambda=\frac{s}{d}$ and $C_{k}\{h\}^{n}$ is the $n$-fold convolution of the function $h$ with itself

$$
\begin{aligned}
& C_{k}\{h\}^{0}=\delta(k) \\
& C_{k}\{h\}^{n}=C_{k}\left\{h, c_{k}\{h\}^{n-1}\right\}
\end{aligned}
$$

where $C_{k}\left\{h_{1}, h_{2}\right\}$ is the convolution of the functions $h_{1}(k)$ and $h_{2}(k)$

$$
C_{k}\left\{h_{1}, h_{2}\right\}=\int_{0}^{k} h_{1}\left(k^{\prime}\right) h_{3}\left(k-k^{\prime}\right) d k^{\prime}
$$

The mean absorption in this case is $\lambda$ times the mean absorption for the individual line

$$
\bar{k}=\lambda \int_{0}^{\infty} h(k) k d k
$$

For large values of $\lambda, h_{R}(k)$ approaches the normal distribution with mean value $\bar{k}$, as given above, and dispersion

$$
\sigma^{2}=\lambda \int_{0}^{\infty} h(k)(k-\bar{k})^{2} d k+\frac{1}{\lambda} \bar{k}^{2}
$$


For the special case of randomly distributed squares, given by Eq. (15), $\sigma^{2}=2 \alpha \mathrm{k}_{0}^{2}$

When $h_{R}(k)$, as given by Eq. (16), is inserted into Eq. (13), the intensity transmission takes on a simple form (Goody, 1964, §4.5.2)

$$
\bar{\tau}\left(p_{1}, p_{2}\right)=\exp (-w / d)
$$

where $w$ is the equivalent width of a single line

$$
w=s \int_{0}^{\infty}\left[1-e^{-k\left(p_{2}-p_{1}\right)}\right] h(k) d k
$$

A similar expression is obtained for the isotropic flux transmission.

Examples of the function $k_{\nu}$ with the corresponding $h(k)$ are exhibited in Fig. 1. For each case, $\bar{k}$ and $\alpha$ were fixed at 2 and .25 , respectively. It is seen that the realistic line shapes such as Lorentz, Doppler, and Elsasser yield U-shaped k-distributions, compared to triangular profiles, which have flat distributions. The square profiles yield $h(k)$ functions which may be considered the opposite extreme to the grey case. The grey case is characterized by a $\delta$-function at a single value of $k$, whereas the square case yields two $\delta$-functions, one at each end of the k-range. From the point of view of "non-grey effects", the k-range is more important than the shape of the $h(k)$ function; the wider the range, the more non-grey the atmosphere. The effect of randomly distributing the lines is to cause the $h(k)$ function to change from concave upward to concave downward. An example is shown in Fig. 2, 
where the formula given by Eq. (16) was applied to series of non-overlapping Lorentz lines to yield the $k$-distribution for randomly distributed Lorentz line profiles (labelled RLO in the figure). This was done for two values of $\alpha: .25$ (corresponding to the case shown in Fig. 1) and 2.0 (a situation in which the lines extensively overlap).

For comparison, the k-distributions for series of regularly spaced Lorentz lines (the Elsasser case, labelled EL in the figure) are also shown. For $\alpha=2$, the $k$-distribution for regularly spaced lines is essentially a 8 - function and is represented by a vertical line at $k=2$, whereas for randomly spaced lines the $k$-distribution has an appreciable width, as shown in the figure.

\section{E. Spectra of Actual. Gases}

In an actual gas, the situation is complicated by the fact that although the line shapes may be assumed identical--and for some bands, the half-width is approximately the same for each line--there is usually a wide range of line strengths within the band. As a result, the highly idealized forms of $h(k)$ do not appear in practice. An example is shown in Fig. 3, where the k-distribution is presented for the portion of the $15 \mathrm{CO}_{2}$ band in the region between 675 and $715 \mathrm{~cm}^{-1}$, calculated at a temperature of $250^{\circ} \mathrm{K}$ and at three different pressures: 1, .1, and .01 atm. An interesting feature of these particular $k$-distributions is that each curve has two parts, each of which fits a power law

$$
h(k) \propto k^{-n}
$$

where $n=.75$ for small $k$ and $n=1.5$ for large $k$. Also, the minimum $k$ is proportional to $p$ and the maximum $k$ is inversely proportional to $p$. These features appear throughout the $15 \mu \mathrm{CO}_{2}$ band and will be the subject of a separate study. 
4. Solution to Atmospheric Structure Equations

The vertical temperature profile and planetary surface temperature are determined from a numerical solution to Eqs. (11) and (12). For the case in which $p_{s}=\infty$--and hence, Eq. (12) and the term involving $B_{s}$ in Eq. (11) are dropped-methods for solving Eq. (11) have been developed by Krook (1963) and Stone (1963). The former method is not easily adapted to the case in which $p_{s}$ is finite. The latter method has been extended by Grossman (1964) to the finite case, yielding solutions for mildly non-grey atmospheres. Both methods are iterative, even for grey atmospheres.

For the present problem, a Newton-Raphson scheme (Scarborough, 1958) was developed. It has the property that it is direct (non-iterative) for grey atmospheres and non-grey atmospheres with a single wave number interval $(0, \infty)$. With multiple wave number intervals--i.e., where the transmission function is different in each wave number interval--the scheme becomes iterative.

The procedure begins with an initial guess $B^{(0)}(p), B_{s}^{(0)}$. (For this purpose, we used the Eddington approximation, in which $B(p)$ is linear in $p$. ) Insertion into Eq. (11) yields an error which is used to generate a correction: $B^{(1)}(p)=B^{(0)}(p)+\Delta B^{(0)}(p)$ and $B_{s}^{(l)}=B_{s}^{(0)}+\Delta B_{s}^{(0)}$. The process is repeated until $\Delta B(p)$ is sufficiently small. For conditions under which the scheme is direct, $\Delta B^{(1)}(p)=0$. In the general situation, as expected with the Newton-Raphson scheme, $\Delta B(p)$ decreases quadratically with each iteration.

To simplify the equations, we assume the variables are in the following units:

$$
\begin{aligned}
p: & p_{s} \\
T: & T_{e} \\
B(T): & \frac{\sigma}{\pi} T_{e}^{4} \\
Q(p): & \frac{\sigma}{p_{s}} T_{e}^{4} \\
k: & 1 / p_{s}
\end{aligned}
$$


Based upon Eqs. (11) and (12) we define two functionals

$$
\begin{aligned}
& F_{1}\left\{B(p), B_{s}, p\right\}= \sum_{i}+4 \bar{k}_{i} B_{i}(p)-B_{s i} \tau{ }_{i}^{10}(p, 1) \\
&\left.-\int_{0}^{p} B_{i}\left(p^{\prime}\right) \tau{ }_{i}^{11}\left(p^{\prime}, p\right) d p^{\prime}-\int_{p}^{1} B_{i}\left(p^{\prime}\right) \tau{ }_{i}^{11}\left(p, p^{\prime}\right) d p^{\prime}\right\}-Q(p) \\
& \mathcal{F}_{2}\left\{B(p), B_{s}\right\}=B_{s}-1+\int_{0}^{1} Q(p) d p-\sum_{i} \int_{0}^{1} B_{i}(p) \tau \tau_{i}^{10}(p, 1) d p
\end{aligned}
$$

and we seek the function $B(p)$ for which

$$
\begin{aligned}
& F_{1}\left\{B(p), B_{s}, p\right\}=0 \quad 0 \leq p \leq 1 \\
& F_{2}\left\{B(p), B_{s}\right\}=0
\end{aligned}
$$

If $B(p), B_{s}$ is a close approximation to the true solution, then we seek corrections $\Delta B(p), \Delta B$ such that

$$
\begin{aligned}
& \mathcal{F}_{1}\left\{B(p)+\Delta B(p), B_{s}+\Delta B_{s}, p\right\}=0 \\
& F_{2}\left\{B(p)+\Delta B(p), B_{s}+\Delta B_{s}\right\}=0
\end{aligned}
$$

The relationship between the $F$ 's and the $B$ 's is linear when there is only one wave number interval, and non-linear when there are more than one wave number intervals. In either case, Eqs. (19) and (20) are linearized by formally expanding the $F$ 's in a Taylor series, retaining only linear terms. This yields two integral equations which are linear in $\Delta B(p)$ and $\Delta B_{s}$ : 


$$
\begin{aligned}
& \sum_{i=1}^{m}\left\{4 \bar{k}_{i}\left[\zeta_{i}(p) \Delta B(p)+B_{i}(p)\right]-\int_{0}^{p}\left[\zeta_{i}\left(p^{\prime}\right) \Delta B\left(p^{\prime}\right)+B_{i}\left(p^{\prime}\right)\right] \tau \tau_{i}^{11}\left(p^{\prime}, p\right) d p^{\prime}\right. \\
& \left.\quad-\int_{p}^{1}\left[\zeta_{i}\left(p^{\prime}\right) \Delta B\left(p^{\prime}\right)+B_{i}\left(p^{\prime}\right)\right] \tau_{i}^{11}\left(p, p^{\prime}\right) d p^{\prime}-\left[\zeta_{i s} \Delta B_{s}+B_{i s}\right] \tau_{i}^{10}(p, 1)\right\} \\
& -Q(p)=0 \\
& \sum_{i=1}^{m} \int_{0}^{1}\left[\zeta_{i}(p) \Delta B(p)+B_{i}(p)\right] \tau_{i}^{10}(p, 1) d p-\Delta B_{s}-B_{s}+1-\int_{0}^{1} Q(p) d p=0
\end{aligned}
$$

where, in our non-dimensional units,

$$
\begin{gathered}
B_{i}(p)=\frac{15}{\pi^{4}} B(p) \int_{\nu_{i-1} / T(p) e^{x}-1}^{\nu_{i} / T(p)} d x \\
B(p)=T^{4}(p) \\
\zeta_{i}(p) \equiv \frac{\partial B_{i}(p)}{\partial B(p)}=\frac{B_{i}(p)}{B(p)}+\frac{15}{4 \pi^{4} B(p)}\left[\frac{\nu_{i}^{4}}{e^{\nu_{i} / T(p)}-1}-\frac{\nu_{i-1}^{4}}{e^{\nu_{i-1} / T(p)}-1}\right]
\end{gathered}
$$

with similar expressions for $B_{s}$.

Numerical solutions to Eqs. (21) and (22) are obtained by replacing the integrals over the variable $p$ by a sum over a set of discrete values of $p$. We choose $n^{+1}$ points in the $p$ interval $(0,1), p_{i}$ for $i=0,1,2, \ldots n$, such that $p_{0}=0<p_{1}<p_{2} \cdots p_{n}=1$. We assume that $B(p)$ is a straight line between each pair of successive points and denote $B_{i}\left(p_{i}\right)$ and $\zeta_{i}\left(p_{i}\right)$ by $B_{i j}$ and $\zeta_{i j}$ ' respectively. Eqs. (21) and (22) then take the form 


$$
\begin{aligned}
\sum_{i=1}^{m}\left\{4 k_{i}\right. & {\left.\left[\zeta_{i j} \Delta B_{i}+B_{i j}\right]-\frac{1}{2} \sum_{k=1}^{i}\left[\zeta_{i k} \Delta B_{k}+B_{i k}+\zeta_{i k-1} \Delta B_{k-1}+B_{i k-1}\right] \tau_{i}^{01}\left(p_{k}, p_{i}\right)-\tau_{i}^{01}\left(p_{k-1}, p_{i}\right)\right] } \\
& -\frac{1}{2} \sum_{k=i+1}^{n}\left[\zeta_{i k} \Delta B_{k}+B_{i k}+\zeta_{i k-1} \Delta B_{k-1}+B_{i k-1}\right]\left[\tau_{i}^{00}\left(p_{i}, p_{k}\right)-\tau_{i}^{10}\left(p_{i}, p_{k-1}\right)\right] \\
& \left.-\left[\zeta_{i s} \Delta B_{s}+B_{i s}\right] \tau_{i}^{10}\left(p_{i}, p_{n}\right)\right\}-Q\left(p_{i}\right)=0
\end{aligned}
$$

$\frac{1}{2} \sum_{i=1}^{m} \sum_{j=1}^{n}\left[\zeta_{i j} \Delta B_{i}+B_{i j}+\zeta_{i j-1} \Delta B_{i-1}+B_{i j-1}\right]\left[\tau_{i}\left(p_{i}, p_{n}\right)-\tau_{i}\left(p_{i-1}, p_{n}\right)\right]$

$$
-\Delta B_{s}-\dot{B}_{s}+1-\int_{0}^{1} Q(p) d p=0
$$

Eqs. (23), (24) are linear with respect to the $n+1 \Delta B_{i}$ values and $\Delta B_{s}$. It can be proven that a solution exists for the case of one wave number interval $(0, \infty)$. This set of equations is solved using a Gaussian elimination scheme with partial pivoting (see, for example, Isaacson and Keller, 1966). Experience with this calculation shows that the truncation error (the difference between the true and computed values) is proportional to $\frac{1}{n^{2}}$. 


\section{Numerical Results}

The numerical solutions presented here are for a homogeneous atmosphere i.e., the pressure and temperature dependence of the absorption coefficient is neglected. The infrared absorption spectrum is assumed to have a grey or non-grey character corresponding to one of the ideal situations discussed in Section 3. The characteristic form of the nongreyness is assumed to hold throughout the infrared spectrum so that we need consider only one wave number interval $(0, \infty)$. The solar heating term $Q(p)$ is taken to be zero - i.e., the atmosphere is assumed to be completely transparent to incident solar radiation.

With these conditions, a grey atmosphere has only one parameter: $\bar{k}$ (in units of $1 / p_{s}$ ) represents optical thickness of the atmosphere at all wave numbers. The radiative equilibrium temperature profile for the grey case is shown in Fig. 4 for $\bar{k}$ ranging from 0.5 to 8 . The results illustrate the well-known greenhouse effect, a property of planetary atmospheres which are transparent to solar radiation but opaque in the infrared. The temperature increases with increasing mass level (or depth) approaching a maximum at the surface, with a temperature discontinuity between the surface and the atmosphere immediately above. As the optical thickness of the atmosphere increases, the temperature throughout increases, except in the vicinity of $p=0$, the top of the atmosphere, where the temperature is practically independent of optical thickness. This last result, that $T(0)$ is practically independent of $\bar{k}$, is a consequence of the approximately one-to-one relationship between the outgoing infrared flux (which is fixed by the value of $T_{e}$ ) and the temperatures in 
the uppermost layer of the atmosphere $(k \leq 1)$; therefore, the temperatures in that layer are a function of $T_{\mathrm{e}^{\prime}}$ more or less independent of optical thickness.

Making the atmosphere non-grey, but keeping the mean optical thickness $\bar{k}$ the same, reduces the temperature at all levels. This is simply the effect of punching holes in the greenhouse while at the same time thickening the walls to keep the mean thickness constant; in that situation the greenhouse is less efficient, inasmuch as the holes permit radiative energy exchange over longer paths. This is illustrated in Fig. 5 for a regular array of square line profiles for which $a=.25$, $k_{1}=0$, and $\bar{k}$ ranges from 0.5 to 8 . Because the outgoing infrared flux is now influenced by temperatures deep within the atmosphere, the temperature at $p=0$ is no longer independent of optical thickness. In this particular case, the temperature at $p=0$ actually decreases with increasing $\bar{k}$, although the temperatures for $p>.25$ increase with increasing $\bar{k}$.

Figure 6 illustrates how the non-grey effects become more pronounced as the atmosphere is made increasingly non-grey. The spectrum is again a regular array of square profiles, with $k_{1}=0$ and $k_{2}$ taking on the values $2,4,10$, and 100; a varies with $k_{2}$ to keep $\bar{k}$ fixed at 2. Near the surface, the non-grey effect saturates qui.ckly for large $k_{2}$, while at $p=0, T \rightarrow 0$ as $k_{2} \rightarrow \infty$. An interesting example of the extreme case where $T=0$ at $p=0$ is described by Goody (1964, Sec. 8.2.2). (In that example, the absorption is represented by a Lorentz profile with halfwidth proportional to pressure, so that at the center of the line $k \rightarrow \infty$ as $p \rightarrow 0$.) 
The temperature in the upper part of the atmosphere is extremely sensitive to $k_{1}$, the absorption coefficient between the lines. This is illustrated for the square case in Fig. 7. The absorption in the center of the lines, $k_{2}$ is fixed at 20 and the absorption between the lines is varied from 0 to 0.2 . The half-width ratio $\alpha$ is approximately 0.05 , varying slightly to keep $\bar{k}$ fixed at 2 . Although the variation in $T / T_{e}$ is no larger than .05 in the lower part of the atmosphere, it is greater than 0.2 in the upper part. The reason why temperatures at small optical depths are particularly sensitive to the absorption between lines is that the temperatures are low and hence, are greatly influenced by the parts of the spectrum that facilitate radiative exchange over longer paths - viz., between the lines, where the absorption coefficients are small.

. The effect of the shape of the line profiles on the temperature distribution is illustrated in Fig. 8 where we compare temperatures for the following line shapes: grey, square, triangular, Lorentz, Doppler, and Elsasser. The parameters are fixed at $a=.25, \bar{k}=2$, and (for $S Q$ and $T R$ only) $k_{1}=0$. These results confirm what one would predict from an examination of the k-distribution functions in Fig. 1. The Elsasser profile, which has the narrowest k-distribution, yields a temperature distribution closest to the grey case, whereas the square profile, which represents the extreme in non-greyness (for fixed $\bar{k}$ and $a$ ), yields temperatures with the greatest departure from the grey case. The Lorentz (non-overlapping) profile is very close to the Elsasser profile, which takes into account the overlapping of the Lorentz lines. The Doppler (non-overlapping) profile, with its fairly sharp peaks and rapid 
drop-off away from the center, is intermediate between the Elsasser and square profiles.

The effect of randomly distributing the line profiles, in contrast to a regularly spaced array, is shown in Fig. 9. The Elsasser profile (regularly spaced Lorentz lines, including overlap) is compared with randomly distributed Lorentz lines for $\bar{k}=2$ and $a=.25$. Although they both have the same line shapes, halfwidths, and number of lines, the random distribution produces deeper and wider gaps, yielding a wider $k$-distribution function (Fig. 2). As a result, the random Lorentz case is more non-grey than the Elsasser case and gives rise to lower temperatures throughout the atmosphere. Also shown in Fig. 9 is a comparison of regularly spaced and randomly distributed square profiles for the same parameters; the effect of randomization is the same as in the Lorentz case, except it is not as pronounced. .

\section{Summary}

\section{A. Method}

A method for treating non-grey radiative transfer problems in homogeneous planetary atmospheres has been presented. The method is general and, except for the assumption that the atmospheric parameters are homogeneous, no approximations are made. It lends itself readily to numerical computation yielding solutions with accuracy limited only by the finite differencing scheme and the spacing of points.

It differs from other methods in that it allows arbitrary dependence of the absorption coefficient on wave number, $k(\nu)$, without requiring tedious line by line 
integration and without the constraints of band models. To derive the transmission functions, the method employs the distribution of $k$-values within relatively wide spectral intervals. This distribution can be specified with much fewer parameters than it is necessary to specify $k(\nu)$, but contains all the information necessary for an exact solution of the homogeneous case.

To solve for the vertical temperature distribution in a planetary atmosphere under conditions of radiative equilibrium, a generalized Newton-Raphson scheme is used. The procedure yields a direct solution (without iteration) when the same k-distribution function applies throughout the spectrum. In the more general case, where the k-distribution function is different in different regions of the spectrum, the scheme is iterative but rapidly converging.

Although the method is restricted to homogeneous atmospheres - a serious limitation for practical applications - it is possible to extend it to limited cases of inhomogeneity. In the homogeneous case, the ordering of $k$-values with respect to wave number is of no consequence to the problem; hence, the $k$-distribution function carries all of the necessary information. In the inhomogeneous case, one cannot discard the ordering information and it would seem necessary to resort to a line by line integration over wave number. However, in at least one important type of situation it seems likely that one can avoid the expense of line by line integration: the case where inhomogeneity is due to pressure broadening. Figure 3 reveals a marked consistency among $k$-distribution functions at different pressures. If one exploits the 
consistency of these patterns, one might be able to handle such problems using the Curtiss-Godson approximation together with k-distribution functions containing pressure dependent parameters.

\section{B. Numerical Results}

Using the method outlined here, numerical experiments were performed to examine the effects of line shapes and band structure on the radiative equilibrium vertical temperature profile in planetary atmospheres. The experiments were performed for a grey atmosphere and non-grey atmospheres with relatively simple absorption coefficient patterns: lines of a particular shape and strength either equally spaced or randomly distributed throughout the spectrum. The line shapes considered were square, triangular, Lorentz, and Doppler, for which the k-distribution functions are shown in Fig. 1 and 2. The results are as follows:

1. In a grey atmosphere, the temperature increases with increasing optical thickness $(\bar{k})$ at all points except at the top of the atmosphere $(p=0)$ where the temperature is practically independent of $\bar{k}$. (Fig. 4)

2. In a non-grey atmosphere, the temperature increases with increasing $\bar{k}$ below a certain height and decreases with increasing $\bar{k}$ above that height. There is no lower limit to the temperature at $p=0$; it approaches zero as the line-widths approach zero for fixed line strengths.

(Fig. 5)

3. Non-grey effects are enhanced as the k-distribution function that characterizes the infrared spectrum is spread out in k-space. The 
more non-grey the atmosphere, the lower the temperature at all points in the atmosphere. Of the cases considered here, the randomly distributed square line profile is the most non-grey and yields the lowest temperatures, for fixed $\bar{k}$ and $a$. The Elsasser model (evenly spaced Lorentz profiles, including overlap) has the narrowest $k$-distribution and yields the highest temperatures of the non-grey cases. The Doppler profile yields results intermediate between the square and Lorentz profiles. (Fig. 6, 8, and 9)

4. The absorption between the lines of the spectrum has a marked effect on temperatures in the upper part of the atmosphere but a small effect in the lower part. Increasing the absorption between the lines raises the temperature in the upper part. In contrast, increasing the absorption at the center of the lines lowers the temperature throughout the atmosphere. (Fig. 6 and 7)

5. For fixed line strengths (equivalent to fixed $\bar{k}$, which was set equal to 2 in these experiments) the temperature in the atmosphere varied over a range which was $12 \%$ near the surface and $25 \%$ near the top of the atmosphere $(p=0)$, depending upon the line shape and on whether the lines were evenly spaced or randomly distributed. (Fig. 8 and 9)

The most significant practical feature of these results is that temperatures near $p=0$ (which correspond to the stratosphere and above) are sensitive to the detailed characteristics of the infrared spectrum of the absorbing gases. The radiative 
heating (absorption) terms depend strongly on the relative distribution of $k$-values between the peaks and the troughs of the spectrum, whereas the radiative cooling (emission) terms depend upon the mean absorption coefficient, $\bar{k}$. Raising the peaks or lowering the troughs, depresses the stratospheric temperature. It is therefore important to determine the absorption spectrum and to correctly treat the non-grey effects when calculating radiative terms in the stratosphere and layers above.

\section{ACKNOWLEDGEMENT}

We would like to thank Professors H. C. van de Hulst and Edward Spiegel for helpful suggestions during the early stages of this work. One of us (A. A.) would also like to thank Professor Yale Mintz for his hospitality during a ten month visit at the Department of Meteorology, University of California at Los Angeles, where part of this work was done. 


\section{REFERENCES}

Arking, A., 1968: Effect of band structure on equilibrium temperatures in planetary atmospheres. Proc. WMO/IUGG symposium on radiation including satellite techniques. WMO Tech. Note 104. Bergen.

Benedict, W. S., and R. F. Calfee, 1967: Line parameters for the 1.9 and 6.3 micron water vapor bands. ESSA Professional Paper 2, U.S. Dept. of Commerce, Washington, 204 pp.

Drayson, S. R., and C. Young, 1967: The frequencies and intensities of carbon dioxide absorption lines between 12 and 18 microns. Tech. Rep., Dept. of Aerospace Engineering, Univ. of Michigan, Ann Arbor, 55 pp.

Feller, W., 1950: An Introduction to Probability Theory and Its Applications, Volume 1. New York, John Wiley and Sons, 419 pp.

Goody, R. M., 1964: Atmospheric radiation. London, Oxford University Press, $436 \mathrm{pp}$.

Goody, R. M., and M. J. S. Belton, 1967: Radiative relaxation times for Mars. A discussion of Martian atmospheric dynamics. Planet. Space Sci., 15, 247 - 256.

Grossman, K., 1964: An iterative solution for the source function in the gray atmosphere in radiative equilibrium. Proc. of the First Harvard-Smithsonian 
Conference on Stellar Atmospheres. Smithsonian Astrophysical Obs. Spec. Rep. 167, 335 pp.

Isaacson, E. and H. B. Keller, 1966: Analysis of numerical methods. New York. John Wiley and Sons, 541 pp.

Kondratyev, K. Ya., 1969: Radiation in the atmosphere. New York, Academic Press, 912 pp.

Kourganoff, V., 1952: Basic methods in transfer problems. London, Oxford Univ Press, 281 pp.

Krook, M., 1963: A perturbation method for non-gray stellar atmospheres. Ap. J.,. $134,863-873$.

Kuhn, W. R., and J. London, 1969: Infrared radiative cooling in the middle atmosphere $(30-110 \mathrm{~km})$. J. Atmos. Sci., 26, $189-204$.

Leovy, C., 1964: Radiative equilibrium of the mesosphere. J. Atmos. Sci., 21, $238-248$.

Murgatroyd, R. J., and R. M. Goody, 1958: Sources and sinks of radiative energy from 30 to $90 \mathrm{~km}$. Quart. J. R. Meteor. Soc., 84, 225 - 234.

Scarborough, J. B., 1958: Numerical mathematical analysis. Baltimore, Johns Hopkins Press, Fourth Ed., 576 pp. 
Stone, P. H., 1963: Approximate integral equations for the temperature in non-gray model atmospheres. Ap. J., 137, $628-640$.

Strom, S. E., and R. L. Kurucz, 1966: A statistical procedure for computing lineblanketed model stellar atmospheres with applications to the F5 IV star procyon. J. Quart. Spectrosc. Radiat. Transfer, 6, $591-607$. 

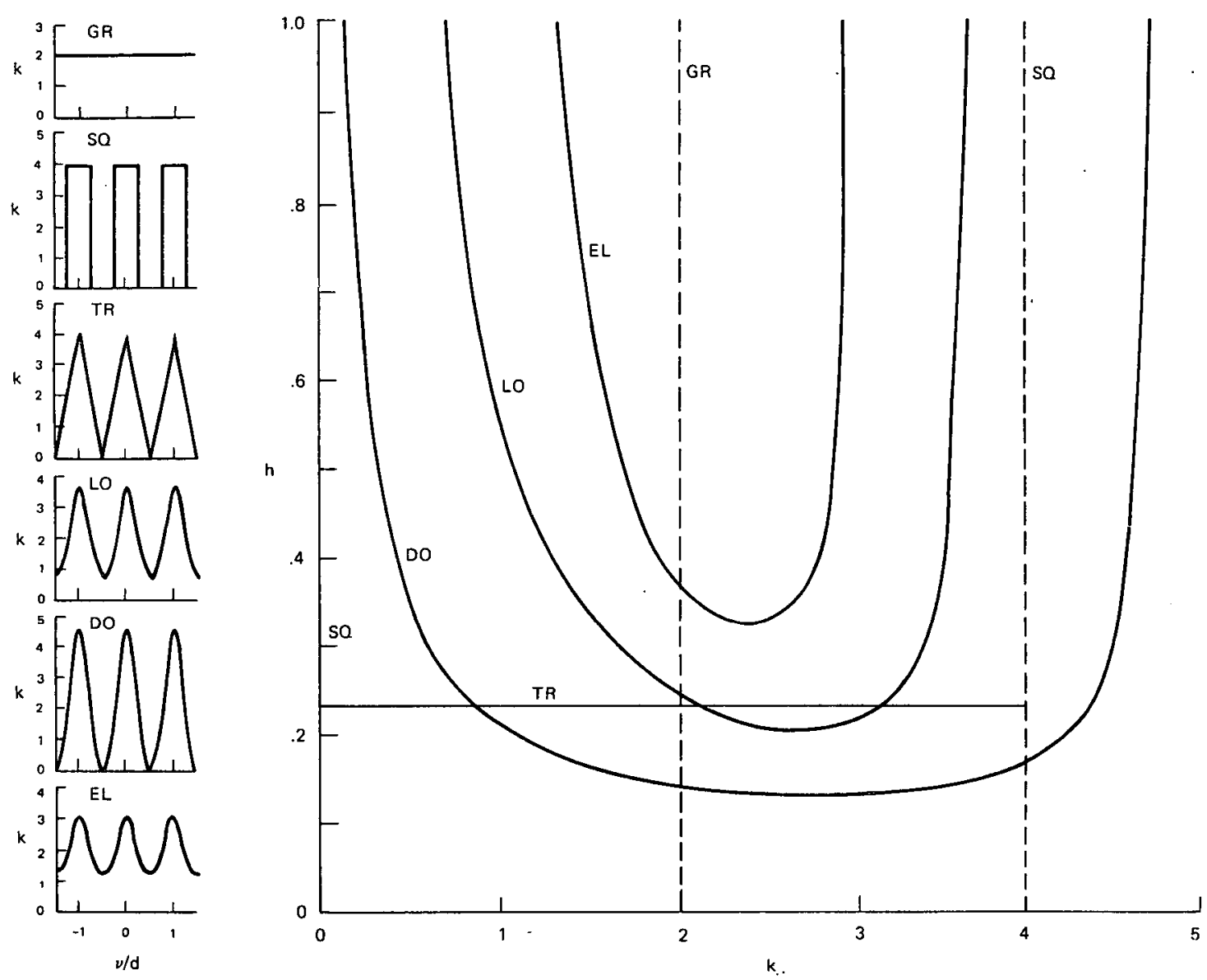

Figure 1. The k-distribution functions for some artificial band models with evenly spaced lines. The curves along the left-hand column show the absorption coefficient as a function of the ratio of wave number to line spacing for each case. The curves on the right are the corresponding k-distribution functions (see text for explanation). $G R=$ grey; $S Q=$ square; $T R=$ triangular; LO = Lorentz; DO = Doppler; $E L=$ Elsasser. (Note that the grey distribution function is a delta function represented by a vertical dashed line at $k=2$, and the square distribution function is the sum of two delta functions, represented by vertical dashed lines at $k=0$ and 4 .) 


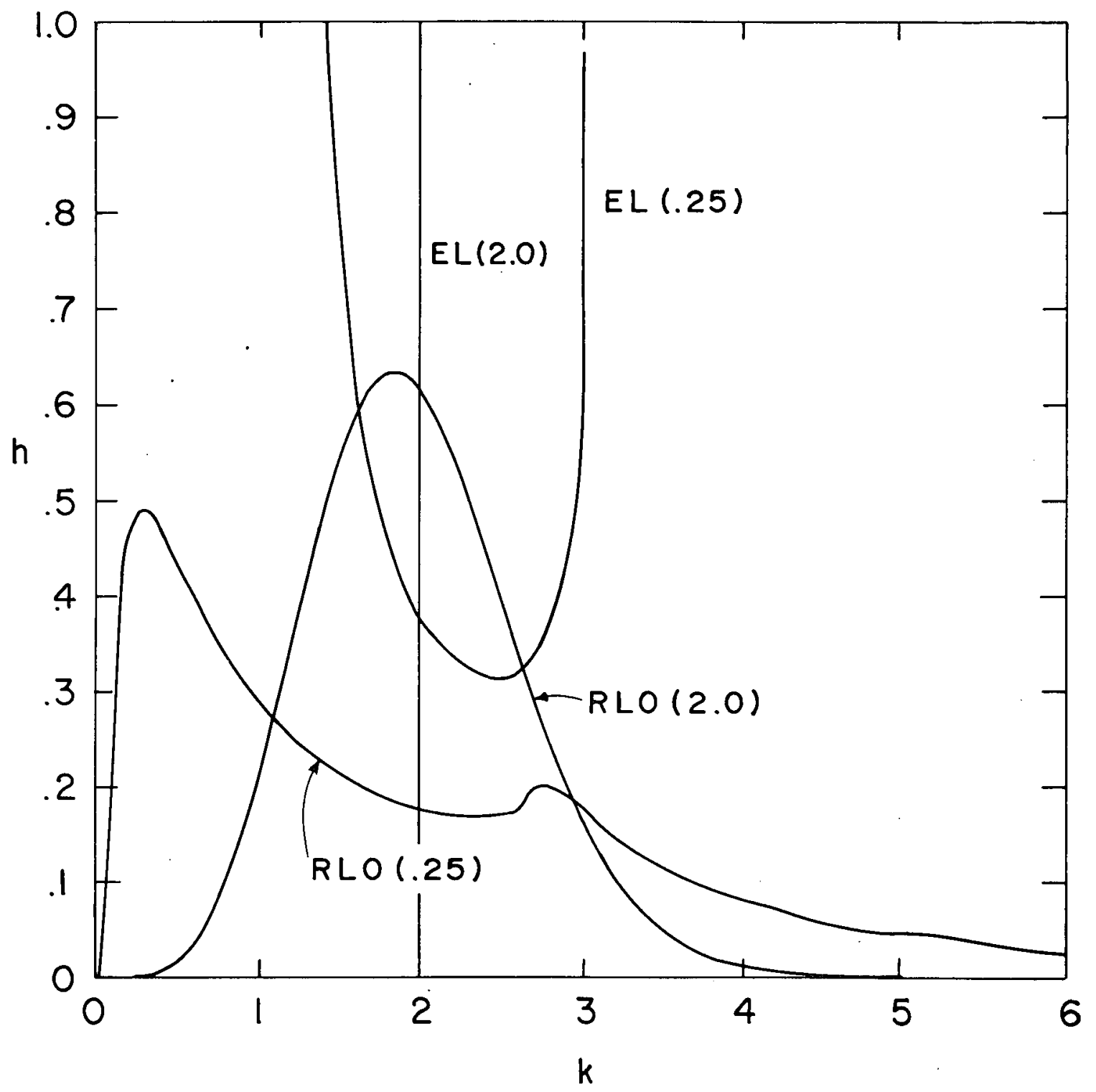

Figure 2. The k-distribution functions for randomly distributed Lorentz line profiles (RLO) and for the Elsasser band (EL), which consists of evenly spaced Lorentz profiles. (Overlap of lines is included in both cases.) They are shown for two values of the ratio of half-width to mean line spacing: $a=.25$ and 2.0. For the Elsasser case with $\alpha=2$, the distribution function is so narrow that it is indistinguishable on this scale from a delta function and is represented by a vertical line of $k=2$. 


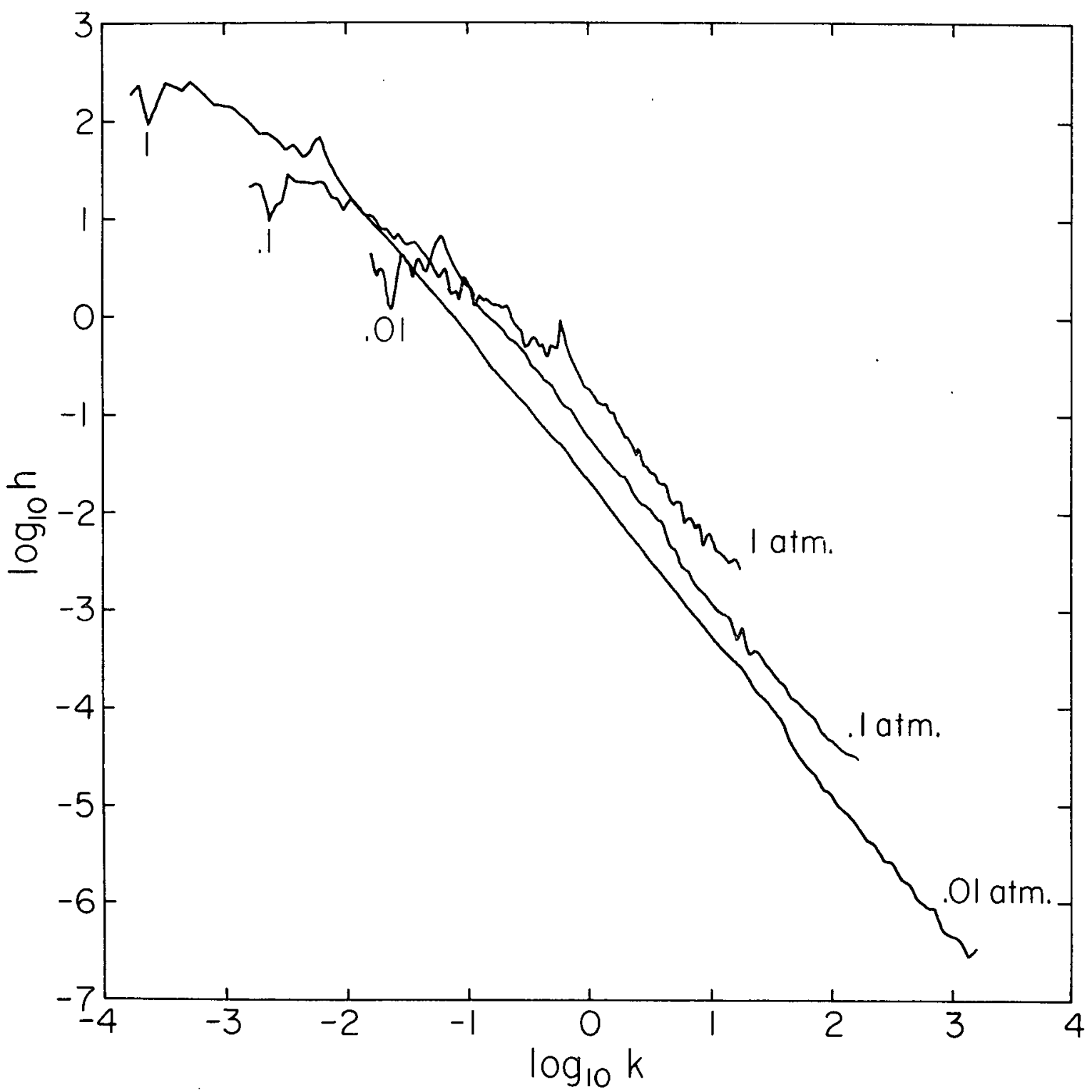

Figure 3. The k-distribution function for the portion of the $\mathrm{CO}_{2} 15 \mu$ band from 675 to $715 \mathrm{~cm}^{-1}$, at a temperature of $250^{\circ} \mathrm{K}$ and pressures of $1, .1$, and $.01 \mathrm{~atm}$. It is based upon Lorentz profiles with a constant half-widih of $.08 \mathrm{~cm}^{-1}$ times the pressure. The line parameters were taken from Drayson and Young (1967). 


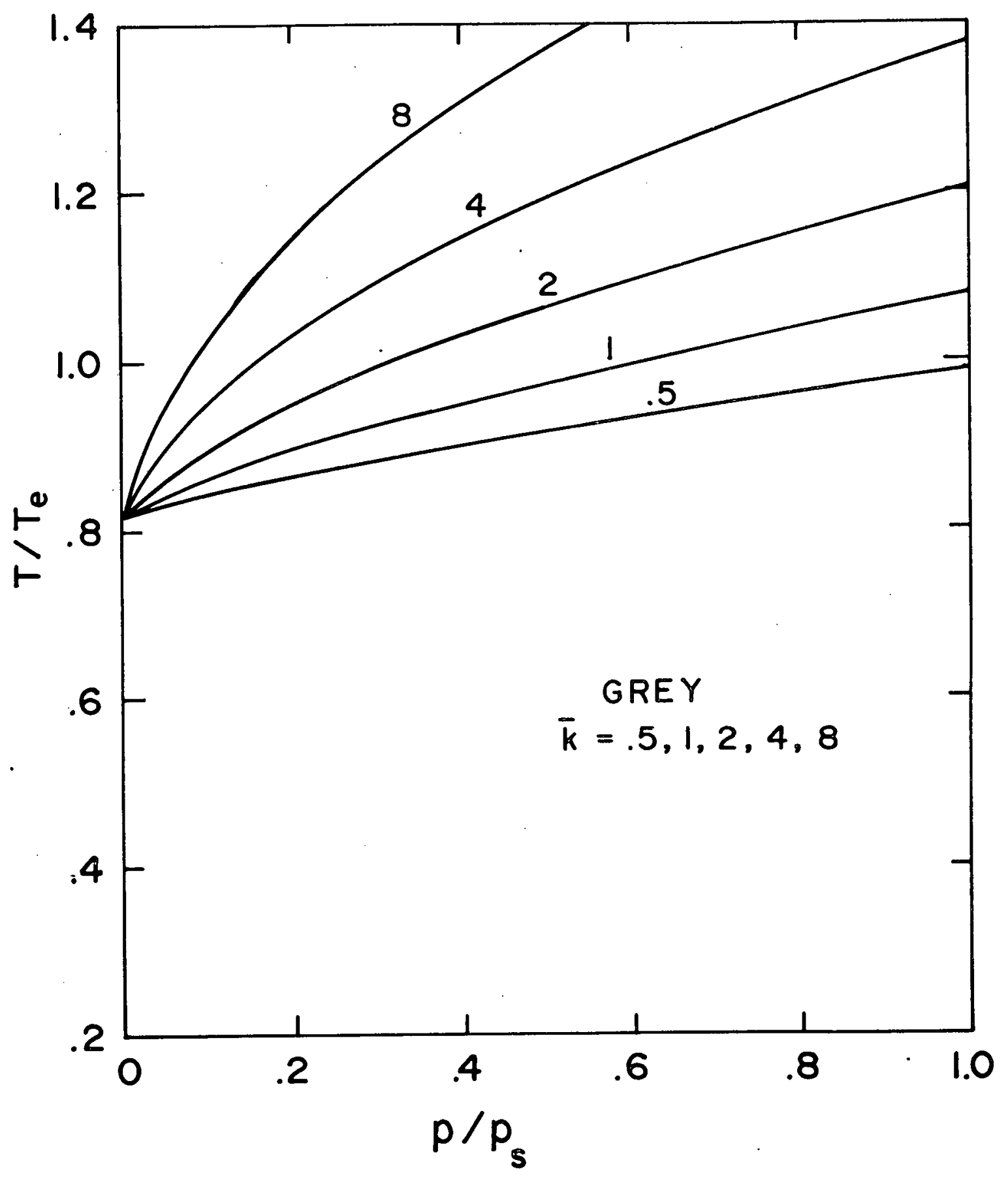

Figure 4. The temperature, in units of the effective temperature, versus pressure, in units of the surface pressure, for a grey atmosphere with optical thickness .5, 1, 2, 4, and 8 . 


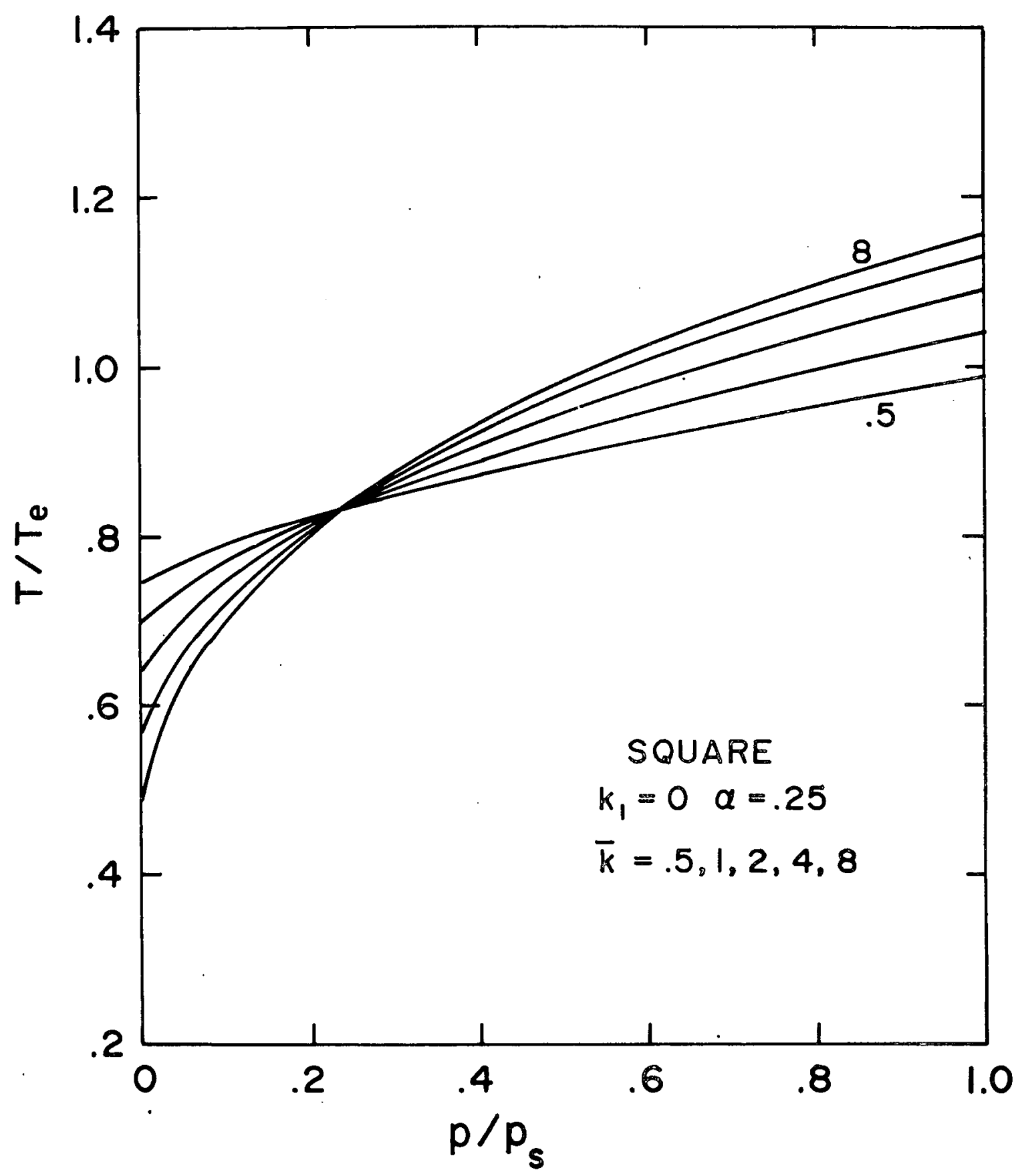

Figure 5. Temperature versus pressure for an atmosphere with evenly spaced square line profiles for the same values of mean optical thickness $(\bar{k})$ as in Fig. 4. $k_{1}$ is the optical thickness of the atmosphere between the lines, $k_{2}$ is the optical thickness within the lines and $a$ is the ratio of half-width to line spacing. 


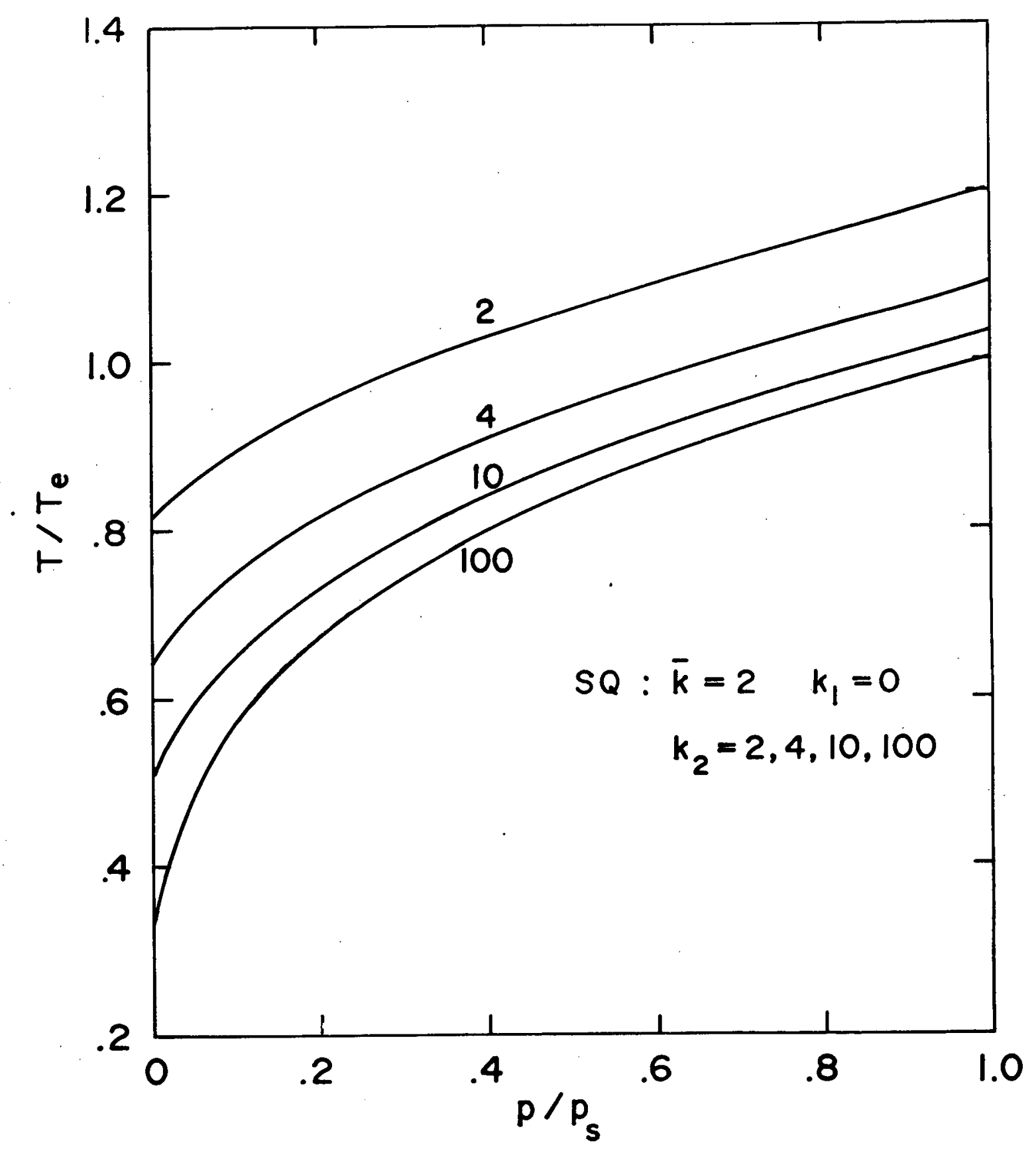

Figure 6. Temperature versus pressure for an atmosphere with evenly spaced square line profiles as a function of $k_{2}$. (See coptions for Figs. 4 and 5 for explanation of symbols.) 


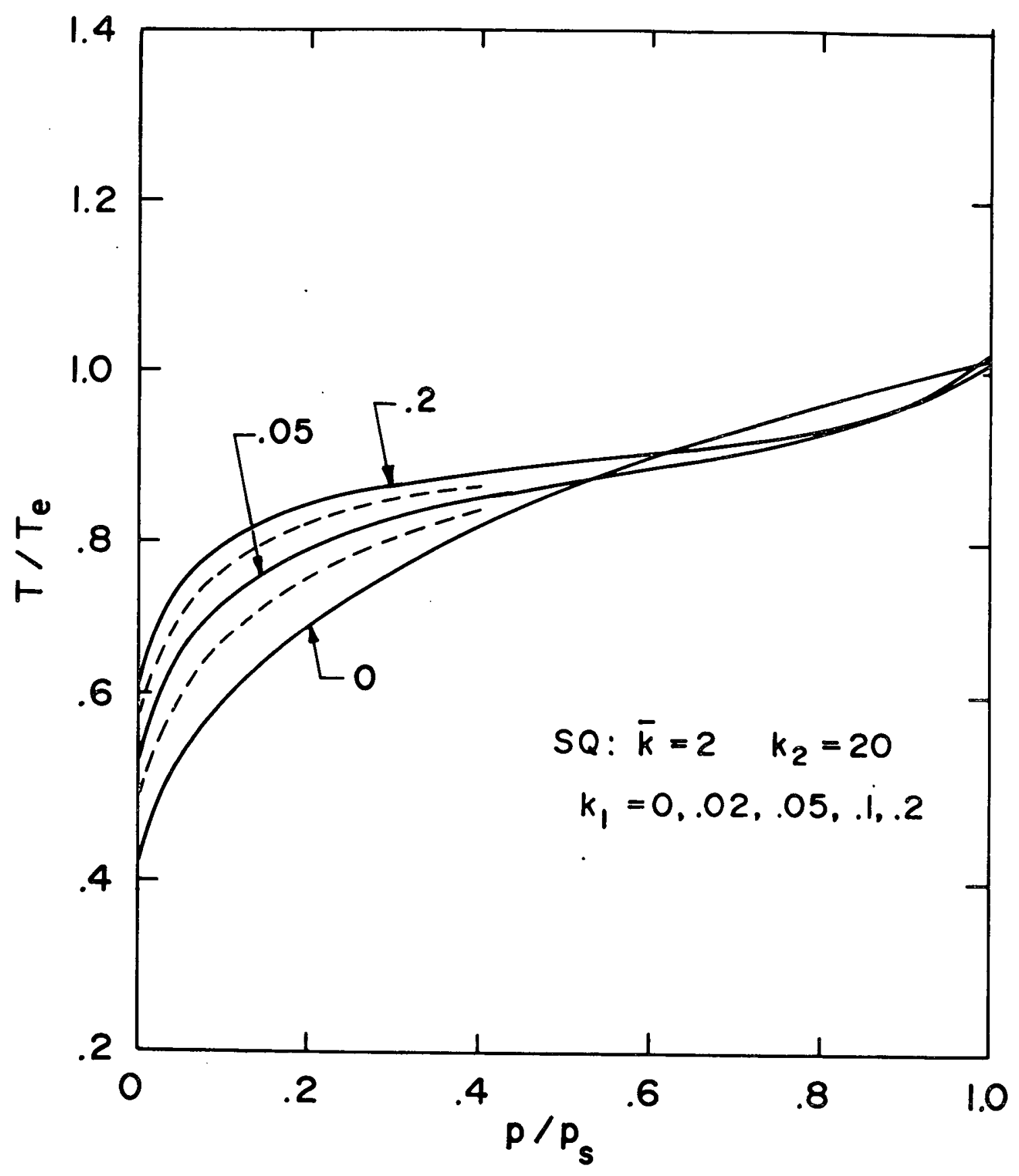

Figure 7. Temperature versus pressure for an atmosphere with evenly spaced square line profiles as a function of $k_{1}$. (See captions for Figs. 4 and 5 for explanation of symbols.) 


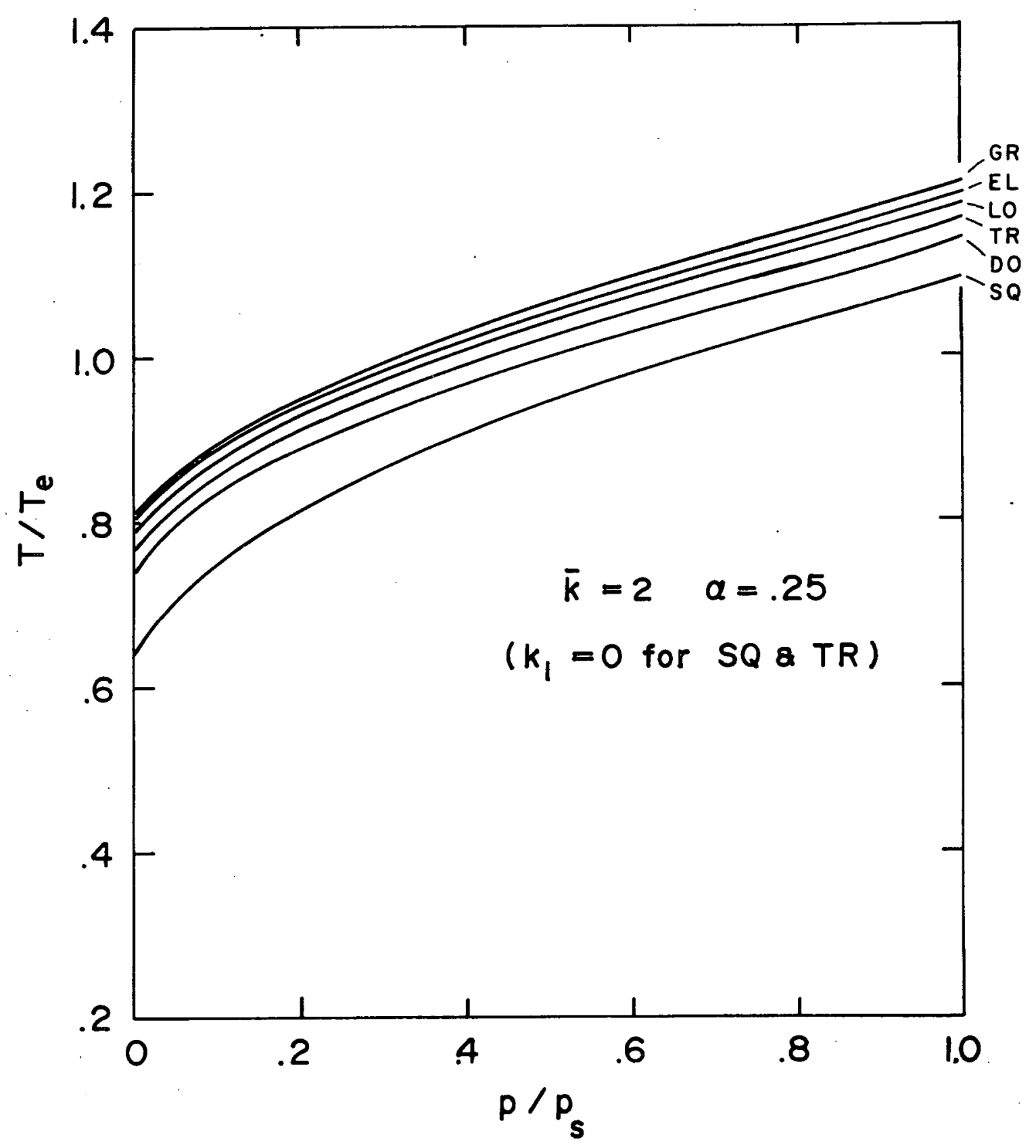

Figure 8. Temperature versus pressure for various band models (see caption for Fig. I for an explanation of symbols) for an atmosphere with mean optical thickness $\bar{k}=2$ and a ratio of half-width to line spacing $\alpha=.25$. 


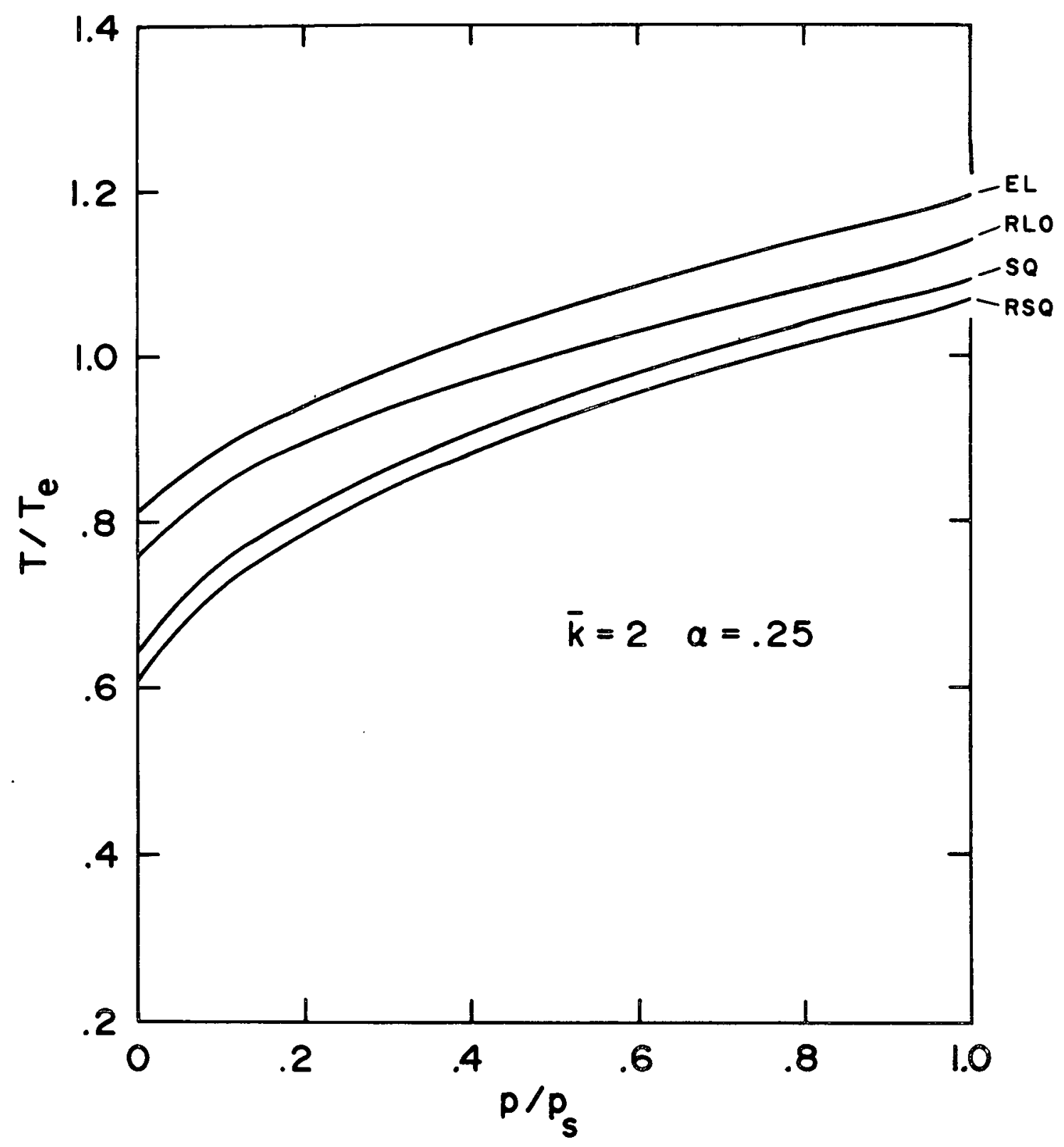

Figure 9. Comparison of temperature versus pressure for atmospheres with randomly distributed lines $(R L O=$ randomly distributed Lorentz line profiles; $R S Q=$ randomly distributed square line profiles) with atmospheres that have evenly spaced lines at the same mean optical thickness $\bar{k}=2$ and ratio of half-width to mean line spacing $a=.25$. $E L=$ the Elsasser band, which consists of evenly spaced Lorentz line profiles; $S Q=$ evenly spaced square line profiles. Overlap of lines is included in all cases. 